\section{POST-GRADUATE CLINICAL DEMONSTRATION}

\author{
Given in the Out-patient Department of St. Mary's \\ Hospital \\ BY
}

\section{ZACHARY COPE, M.S. LOND.}

The cases that I am going to demonstrate are such as have come in the ordinary course of seeing out-patients, and will therefore not present a homogeneous group.

First there are three injuries-one of the thumb, two of the ankle.

\section{Injury to the Thumb.}

Here is a lady, middle aged and rather stout, who states that a few days ago she fell with her left hand underneath her. Ever since the accident she has had pain at the base of the thumb and a certain amount of swelling and tenderness of the thenar eminence. Movement of the thumb is rather painful; the wrist is normal. Now on examining this lady's thumb it will be noticed that there is no swelling over the phalanges, but there is a definite circumscribed bruise on the dorsum of each phalanx at its base. There is no tenderness at these areas, the only tenderness is found on pressure at the base of the thenar eminence. As already mentioned the lady can move the thumb but with a little pain.

Now here we have a clear case in which the symptoms definitely fix the seat of injury. There is no need to invoke the aid of a radiogram, though for confirmation of the diagnosis it is perhaps wise to inspect an $X$ ray photograph. The diagnosis is an impacted fracture of the base of the metacarpal bone of the thumb. The reasons for the diagnosis are as follows.

The two separate bruises on the back of the thumb correspond to the sites of insertion of the extensor tendons. These circumscribed ecchymoses without tenderness indicate that blood has travelled along the tendons and come to the surface at their insertion. The injury must therefore be close to the bone for the tendons lie close to the first metacarpal. The circumscribed tenderness and swelling at the base of the metacarpal bone, taken together with these remote bruises, indicate a fracture of the bone at the site of tenderness. The free movement of the thumb proves that the fracture is impacted. The $\mathrm{X}$ ray exactly bears out the diagnosis.

This particular fracture is usually associated with a certain amount of backward displacement of the main part of the shaft of the metacarpal, and is known as Bennett's fracture. I do not remember to have seen one in a woman before. It nearly always occurs in a man and the great majority of those which I have seen have been in boxers. The blow known as the round-arm punch, in which the striker swings his arm and strikes the opponent'क jaw with the radial part of the hand is generall responsible for the lesion. This lady denies tha she has been boxing, but in any case I cannot credither statement that she fell with the palm of the hand towards her body; the only conceivable way in which this accident could have occurred is that she fell palm downwards against some harf substance-i.e., the ground. Since there is n appreciable displacement in this case all that is necessary is to splint the thumb for a week or two and prescribe massage at an early date.

\section{Two Cases of Ankle Injury.}

The next patient is an old lady. whose right food slipped under her a few days ago. Since then she has felt severe pain on the outer aspect of the ankle and cannot walk properly.

On examination there is no swelling or tendernesit over the inner aspect of the ankle. A Pott's fractures therefore, can be excluded. On the outer aspect there is œdema over the external malleolus and round about it for some distance. There is ver\&s little bruising, but as the finger is pressed gently over this area she winces slightly over the midd of the malleolar process. That is the maximur site of her pain. In front of the malleolus she is not so tender.

From these facts the diagnosis becomes clear? There are only two common lesions in this regito produced by a wrench of the foot. One is a fracture of the external malleolus (or just above it Secondly, a rupture of the anterior bundle of fasciculus of the external lateral ligament of th ankle. Now this anterior bundle runs from the front of the malleolus forwards to the astragalus and when it is ruptured-a very common occurrence - the maximum tenderness is always about an ine in front of the external malleolus. Such is not the case here. The maximum tenderness, which 通 nearly every injury represents the site of the lesion, is in this case over the malleolus, and we must therefore diagnose a fracture of the malleolus The official report on the $X$ ray photograph says, "probably a fracture of the external malleolus.o" I think the fracture is a certainty, but it is unusua in being almost vertically through the outer pa of the malleolus.

The treatment is to put the foot and leg in divided plaster-of-Paris splint which can be taket off for massage, which is needed almost from thes first.

The second case of ankle injury is a lady of middre age who has severely wrenched her ankle. Tlise whole ankle is swollen and there is great bruising extending right up the leg and down to the heet. As the ankle is palpated there is great tendernes over the inner aspect, but greater tenderness on pressure over the external malleolus. The bruising is again noticeable. Too little attention is paid to 
the distribution of bruising as an indication of the site of fracture. A rule which is of fairly general application, but which I have never seen mentioned in a text-book is this: When bruising comes to the surface at a distance from the site of the applied trauma, the centre of the circle of bruising will approximately indicate the site of injury. Here is an example: When a person falls on the shoulder and fractures the surgical neck of the humerus the ecchymoses will be seen not so much over the deltoid but below its insertion and above the clavicle and acromion. In this case there is not much bruising over the malleolus, but a great deal below it, above it, and, in fact, all round it. The chances are, then, that the malleolus is fractured and the $X$ ray shows that such is the case, for a transverse fracture can easily be seen in the bone. The tenderness over the internal malleolus makes it fairly certain that the internal lateral ligament has been severely torn. We are therefore dealing with a modification of Pott's fracture. There is but slight displacement, and if a plaster splint is supplied and massage commenced early the patient should get a very useful foot again.

\section{InJURY TO THE UlNar NeRve.}

Here is another type of injury : a young man whose forearm was cut by glass. At the time of the injury the ulnar nerve was found to be severed. It was sutured; but, as might be expected in an accidental wound, suppuration ensued. This happened five weeks ago. The case is sent to me for an opinion as to whether the wound should be reopened to make certain that the nerve-suture is intact. The question can easily be answered. First it can be laid down as a general principle that when suppuration has occurred in a wound it is unwise to perform any plastic operation within at least six months from the time of infection. During the war, when we did a good deal of bone-grafting for the repair of gaps in the jaw, we found by experience that it was unwise to go on with the operation if any trace of sepsis was discovered, and wise to allow a period of about six months between the occurrence of sepsis and a secondary grafting operation. Presumably microbes become less virulent or die out with lapse of time. Now I consider in this case it would be unwise to attempt secondary suture of the nerve within six months of the time of accident-i.e., for another five months. But there is another reason why operation is inadvisable. Though the patient cannot adduct the thumb or separate the fingers properly, thus showing the palm muscles supplied by the ulnar nerve are still paralysed, yet pressure and light touch can be faintly appreciated over parts of the little finger and adjacent ulnar border of the palm. It is clear, therefore, that even less than two months after the accident some of the sensory functions of the nerve are returning. It would therefore be the height of folly to interfere with this process. It would appear that repair is taking place in spite of the suppuration.
Loose Fibro-Cartilage of Temporo-Maxillary JoINT.

The next case is that of a young lady who suffers from the unfortunate drawback of not being able to open her mouth more than about an inch. She is young and in other respects perfectly healthy. There is no sign of tetanus nor any obvious reflex cause for trismus. She can eat fairly well, but when she attempts to bite an apple--i.e., opening the mouth rather wide-she experiences pain in the left temporo-maxillary joint. This condition has only troubled her for three weeks, but is making her life a burden. Prior to its onset she used frequently to have what she termed a clicking of the jaw. Now many people can click their jaws and I see a good number of patients to whom this clicking is a nuisance. Sometimes the click is quite audible to others. Often the patient is self-conscious about it. What is the reason of the click? It will be remembered that between the bony surfaces of each temporo-maxillary articulation there is a thin fibro-cartilage which moves when the mouth is opened. Sometimes this fibro-cartilage is unduly mobile, and this it is which causes the click. In this patient on the left side the fibro-cartilage must have got jammed between the joint surfaces, causing a limitation of movement similar to that which occurs so commonly in the knee. Since it is causing her so much discomfort, I have advised her to come into hospital and have the affected fibro-cartilage removed. In those cases in which there is no locking but only a clicking, removal is unnecessary, and the condition should be as far as possible ignored.

\section{Severe Head Injury.}

This case is that of a little boy upon whom I operated more than a year ago for a severe head injury. He is only about 6 years old. When he was admitted he was suffering from severe concussion. He remained unconscious and symptoms of compression developed. When there was evidence of persisting pressure upon the mid-brain as well as the cerebral hemispheres I decompressed his skull to ward off the impending fatal issue. I found a fissured fracture of the vault of his skull, and when I had removed an area as large as the palm of the hand the brain substance bulged out to a great degree, showing that there was extensive cerebral œdema. Incidentally, one should remember that compression of the most serious kind may follow from this odema, even though there be but slight hæmorrhage. Improvement occurred immediately after the operation, and everyone who saw the case would admit that the decompression saved the boy's life. But it left a big gap in his skull, through which the pulsations could easily be seen and felt. These skull-gaps are rather inconvenient, for the brain substance may be injured by blows which would be of no consequence on the bony skull. (Of course, there was no bulge to be seen a few weeks after the operation, for the cerebral œdema subsided gradually.) There are three ways 
of protecting such a gap in the skull. One is to wear a protective splint outside the scalp. Another way is to put a metal or celluloid cap over the gap in the subcutaneous tissues. The third way is to fill in the gap by grafting cartilage. In this lad's case I first tried grafting cartilage and succeeded in filling in a considerable portion by cartilage taken from his iliac crest. But the protection given was not to my mind sufficient, so a few months back I placed a celluloid cap outside the dura so as to protect the brain substance. In addition you will see that the boy's mother likes him to wear a protective splintcap. I do not, as a rule, like introducing foreign substances such as celluloid, and have before now had to remove them because they caused irritation, but in this case I thought it worth trying.

\section{Hip Disease.}

A lad of 16 came to me recently because of a limp and pain in the left knee. He did not complain of pain in the hip ; but, of course, pain in the knee often results from disease of the hip. What more natural when the same nerves supply both joints? This boy has only ailed for three or four weeks, but already presents definite indications which must be ascribed to tuberculosis of the hip. The points which indicate this I will demonstrate. First, as the boy lies on the couch there is rather a big gap between the lumbar vertebræ and the couch-i.e., there is lordosis, but when I raise the right knee and flex fully the right hip so as to undo the lordosis and bring the lumbar spine on to the couch, the left knee rises up spontaneously owing to flexion of the left thigh. This flexion of the left thigh disappears again if the right lower limb is brought down to the couch, but simultaneously the lordosis reappears. In other words, the lordosis masks the permanent flexion of the left thigh. Again, on looking at the anterior superior spines it is noticed that the left is lower than the right, and if a line is drawn from the umbilicus through the symphysis pubis it crosses the right thigh instead of going midway between the two legs. This means that the right thigh is adducted, the left thigh abducted, and the left side of the pelvis tilted down. The left foot projects an inch below the right one, but when the limbs are placed symmetrically to the pelvis it is seen there is no real shortening. On attempting to move the left hip it is noticed that the pelvis (tested by the anterior superior spine) moves with each attempted movement. Here, then, we have flexion and abduction at the left hip, limitation of all movements at the joint and apparent lengthening of the left lower limb, with compensatory lordosis of the lumbar spine. This represents the first or early stage of tuberculosis of the hip. In view of the comparative recent date of the symptoms, three weeks, one would not expect to find much radiological confirmation, but the $X$ ray shows that there is already considerable erosion of the acetabulum.

This is a very typical case, and there is no difficulty in diagnosis. Acute coxa vara, congenital dislocation of the hip, pseudo-coxalgia, or Perthes? disease, coxa valga, rheumatic arthritis-all these in this case can easily be excluded. It is not necessary to have any specific test for tuberculosi carried out, for in such a case the clinical signs an symptoms are much more reliable than any test: which the laboratory as yet can furnish. As t $\overrightarrow{\text { fi }}$ treatment, the ideal treatment is for the lad to entef a sanatorium and rest in an apparatus which wi年 immobilise both hips in abduction, but until he cat. be got away we intend to immobilise the left his as far as possible by means of a Thomas hip-splint This is admittedly a second best until the ideaw treatment can be provided.

\section{ON THE IMPORTANCE OF POST-GRADUATE TRAINING}

BY

Most of us will readily agree that the educatio which the medical man receives after graduatiog is of vital importance to him in his future caree During his undergraduate period he always has in front of him the necessity for passing examinations at various stages on subjects, many of which apparently play a trivial and unimportant partig his general usefulness to the community. The method follows in a natural sequence of events of that adopted by his preparatory school-master an later by his public school-masters. Perhaps the prominent fault of all early education is that the pupil is chiefly made to learn what the educationgli authorities consider to be facts. Fortunately, there is a growing disposition on the part of modt teachers to train pupils to think rather than store their minds with information, some of whic at least, subsequent research shows to be incorrect. Medical undergraduates in particular should taught to observe for themselves and to think for themselves, for all past experience shows that mene authority is a feeble guide to truth, and, further,, doctor's whole work consists in observing the congplaints, the symptoms, and the physical signs of his patients, and, from what he observes, deducing th correct diagnosis and the most suitable treatmerte. It is admittedly difficult to frame examinations so to bring out a candidate's power of mind rather thay his mere memorising faculty, but the best examiness try to attain this object, and certainly examinations, although still very far from perfect tests, are les⿱乛⿻上丨⿹ objectionable than they used to be, and mope attempts are being made to appraise the mind arid even the character of the candidate. Teachers, tod, are trying to train the student's mind and to shogiv him the "reason why" for things, and sorfte teachers, as for example Sir Arthur Keith, succeeg 